\title{
Correction to: Grotta Romanelli (Lecce, Southern Italy) Between Past and Future: New Studies and Perspectives for an Archaeo-geosite Symbol of the Palaeolithic in Europe
}

\author{
R. Sardella ${ }^{1} \cdot$ D. A. lurino ${ }^{1} \cdot$ B. Mecozzi ${ }^{1} \cdot$ D. Sigari ${ }^{2,3} \cdot$ F. Bona $^{4} \cdot$ L. Bellucci $^{5} \cdot$ \\ M. Coltorti ${ }^{6} \cdot$ J. Conti $^{1} \cdot$ G. Lembo $^{2} \cdot$ B. Muttillo ${ }^{2} \cdot$ I. Mazzini $^{7}$ (D)
}

Published online: 26 July 2019

(C) The European Association for Conservation of the Geological Heritage 2019

\section{Correction to: Geoheritage \\ https://doi.org/10.1007/s12371-019-00376-z}

The original version of this article unfortunately contained a mistake. The following acknowledgement has been missing.

Grandi Scavi 2016, 2017, 2018 of Sapienza, University of Rome (resp. Raffaele Sardella) is acknowledged for funding.

The online version of the original article can be found at https://doi.org/ 10.1007/s12371-019-00376-Z

I. Mazzini
ilaria.mazzini@igag.cnr.it
R. Sardella
raffaele.sardella@uniroma1.it
D. A. Iurino
dawid.iurino@uniroma1.it
B. Mecozzi
beniamino.mecozzi@uniroma1.it
D. Sigari
sgrdra@unife.it
F. Bona
fabgeo@libero.it
L. Bellucci
luca.bellucci@unifi.it
M. Coltorti
mauro.coltorti@unisi.it
J. Conti
jacopo.conti@uniroma1.it

G. Lembo

lmbgpp1@unife.it

B. Muttillo

brunella.muttillo@unife.it

1 Dipartimento di Scienze della Terra, Università di Roma, P.le Aldo Moro, 5, 00185 Rome, Italy

2 Dipartimento di Studi Umanistici, Università degli Studi di Ferrara, C.so Ercole I d'Este 32, 44100 Ferrara, Italy

3 Geosciences Center, University of Coimbra-Pólo II, Rua Sílvio Lima, 3030-790 Coimbra, Portugal

4 Dipartimento di Scienze della Terra Ardito Desio, Universitàdi Milano, Milan, Italy

5 Museo di geologia e Paleontologia, Sistema Museale di Ateneo, Università di Firenze, Via Giorgio La Pira 4, 50121 Firenze, Italy

6 Dipartimento di Scienze Fisiche, della Terra e dell' Ambiente, Università di Siena, Via Laterina 8, 53100 Siena, Italy

7 CNR, IGAG, Area della Ricerca di Roma 1-Montelibretti, Via Salaria Km 29,300, 00015 Monterotondo, Rome, Italy 